\title{
Method of Preventing Cutting Edge Failure of Hob Due to Chip Crush*
}

\author{
Masaharu KOMORI**, Masaoki SUMI*** and Aizoh KUBO**
}

\begin{abstract}
Dry hobbing has great advantages such as its environmental friendliness and its ability to reduce manufacturing cost. Dry hobbing, however, often causes failures of the cutting edge of a hob or problems on the surface quality of the tooth flank of a manufactured gear. The pinching and crushing of generated chips between the cutting edge of a hob and the tooth flank of a work gear is considered to be a major cause of such problems. A simulation method of cutting using a hob was reported. In this report, the simulation is applied to solving the industrial problems of dry hobbing. The simulation clarifies the mechanism of cutting edge failures. The "distance of single-edge cutting" of a hob tooth is proposed to be an index of pinching and crushing of chips. Factors influencing this index are investigated and fundamental countermeasures against pinching and crushing are shown. This method has been applied to solving problems in the mass production of automotive gears, and good results were obtained.
\end{abstract}

Key Words: Gear, Cutting, Chip, Dry Hobbing, Chipping, Hob Damage

\section{Introduction}

Dry hobbing is more environmentally friendly than the conventional method using cutting oil (wet hobbing). It has great advantages in increasing productivity and decreasing manufacturing cost ${ }^{(1),(2)}$. Dry hobbing, however, often causes problems such as the chipping of the cutting edge of a hob or the coarse tooth flank of a manufactured gear. Such problems are not serious in the wet hobbing. The pinching and crushing of chips generated between the cutting edge of a hob and the tooth flank of a work gear is considered to be a major cause of such problems.

Three types of chip crush between the cutting edge of a hob and the tooth flank of a work gear are possible: (1) crush during chip generation, (2) crush of chips flying after its generation, (3) crush of chips stuck on the hob tooth after a hob rotation ${ }^{(3)}$. The problem generated by item (2) can be solved by equipping the hobbing machine with air blower. The same remedy or/and the selection of an adequate coating and material of the hob can improve problems concerning item (3). However, a remedy

* Received 20th May, 2004 (No. 02-0993)

** Department of Precision Engineering, Kyoto University, Yoshidahonmachi, Sakyo-ku, Kyoto-shi, Kyoto 6068501, Japan. E-mail: komorim@ @rec.kyoto-u.ac.jp

*** Gifu Gear Manufacturing Co., Ltd., 2-2-2 Usa-minami, Gifu-shi, Gifu 500-8367, Japan for problems concerning item (1) has not been found because of complex mechanism of hobbing and chip generation.

A simulation method of evaluating the conditions of chip formation and the clearance between the cutting edge of a hob tooth and the tooth flank of a work gear was proposed, where the trace of each cutting edge of the hob relative to the work gear is calculated. The simulation clarifies the mechanism of chip crush between the cutting edge of a hob and the tooth flank of a work gear and enables the evaluation of probability of chip crush. The influence of gear dimensions, hob dimensions and cutting conditions on chip crush is investigated by the proposed method. A method of preventing chip crush is clarified and its effect is confirmed by applying it to actual industrial hobbing.

\section{Observation of Dry Cut Hob Failure and Gener- ated Chips}

Figure 1 shows the typical chipping failure of the cutting edge of a hob observed when a right-hand helical gear (module 2.75, pressure angle 20 degrees, number of teeth 62, helix angle 30 degrees and addendum modification factor -0.6) is dry-cut using a right-hand carbide hob (4 threads and outer diameter $100 \mathrm{~mm}$ ) in climb cutting ( $2.5 \mathrm{~mm} / \mathrm{rev}$.). Figure 2 shows some examples of the chips generated in the same hobbing process: Chips on the right side in (a) and (b) shows the original shape generated and 


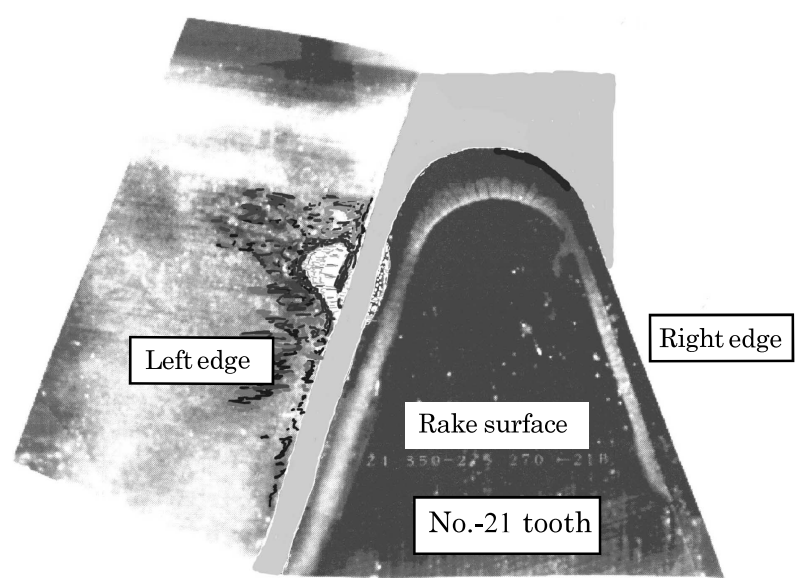

Fig. 1 Chipping of cutting edge of carbide hob under dry cutting

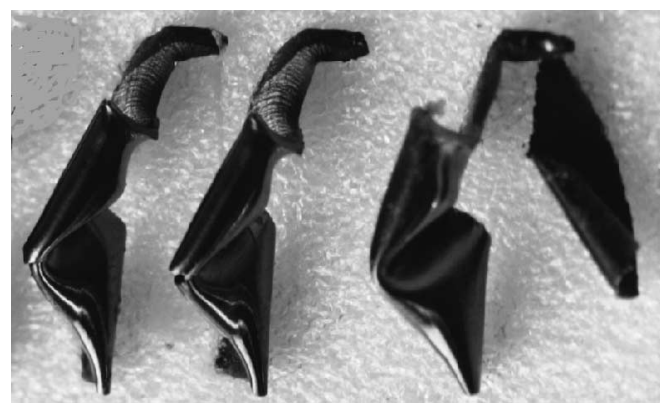

(a) Chips bent after the formation

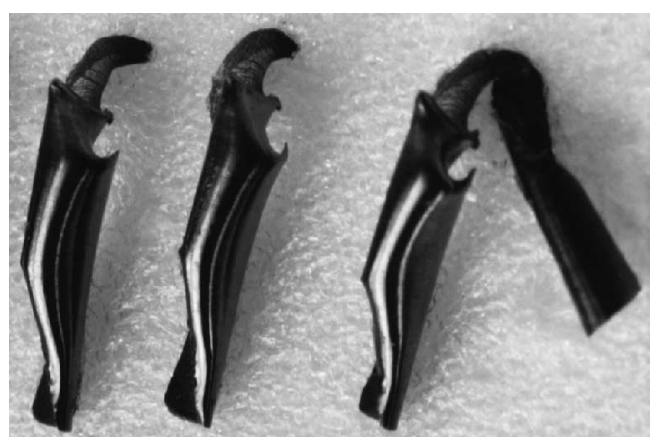

(b) Same kind of chips as that of (a), but not bent after their formation

Fig. 2 Bent or crushed chips observed under dry cutting

the others are separated part from the original chips during this observation. Chips in (a) resemble those in (b) in size and shape, but each chip in (a) is bent. It is thought that these chips in (a) are pinched and crushed between the cutting edge of the hob and the tooth flank of the work gear. Many examples of such bent chips are found in piles of discard chips. This indicates that this chip crush does not occur by accident. Rather, a repetitive definite mechanism of chip crush must exist in hobbing.

The chips in Fig. 3 have a cutter mark on the surface at the same part. These chips are also considered to be crushed between the cutting edge of the hob and the tooth flank of the work gear during hobbing.
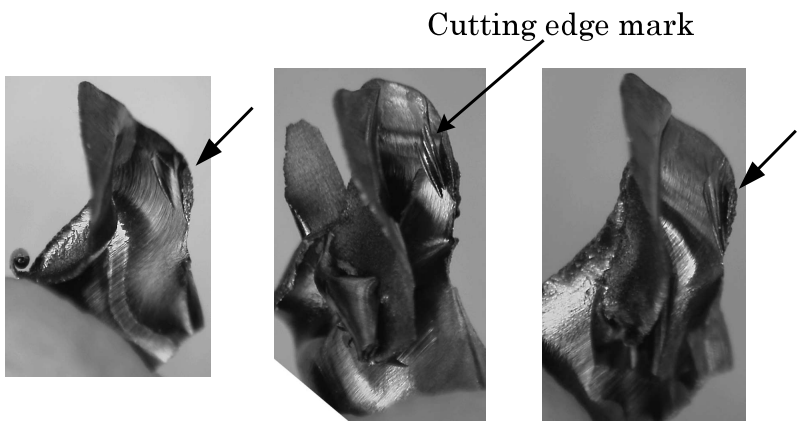

Fig. 3 Cutting edge marks found in the same position of chips, observed under dry cutting

The above findings show that there is a steady mechanism that causes chip crush between the cutting edge of a hob and the tooth flank of a work gear. Thus, the chipping of the cutting edge of the hob in Fig. 1 is probably caused by chip crush.

\section{Comparison of Simulation Result and Actual Hob- bing}

It is possible to evaluate the changing state of chip generation and of clearance between the cutting edge of a hob and the tooth flank of a work gear by the proposed simulation $^{(4)}$. This is worked out by comparing tooth groove shape before 1 revolution of the work gear, the trace of the previously acting cutting edge, and the trace of the object cutting edge. In such a simulation, a cutting edge is expressed by a series of points and the trace of the cutting edge is composed of the trace of those points. A hob tooth for generatng the center of the tooth groove of a work gear is defined as No.0 tooth, those acting before No.0 tooth have a negative number, and those acting after No.0 tooth have a positive number. Looking at the hob tooth tip on the upper side, the side edge located on the left is called the left edge, and vice versa, cf. Fig. 1.

Figure 4 shows the simulation result for the cutting edge of the No.-21 tooth of the hob shown in Fig. 1, which is expressed by traces on each normal-to-axis slice of the work gear, as shown in Fig. 5 (b). The object tooth groove is considered to be located on the far side of the work gear body and that is observed from a viewpoint below the work gear as shown in Fig. 5 (a). The layout of the trace on each slice for different positions is so arranged in Fig. 4 for easy understanding that the tooth groove seems the straight though the object gear is helical. In Fig. 4, the inclined line below each small figure on the slice at definite position indicates tip circle of the work gear and the vertical line shows the trace of each point on the cutting edge of the No.-21 hob tooth. The thin broken curve indicates the tooth groove shape formed before 1 revolution of the work gear, the thin black curve the trace of the cutting edge of the No.-22 hob tooth acting before the No.-21 cutting edge, and the wider thick line the trace of the No.-21 


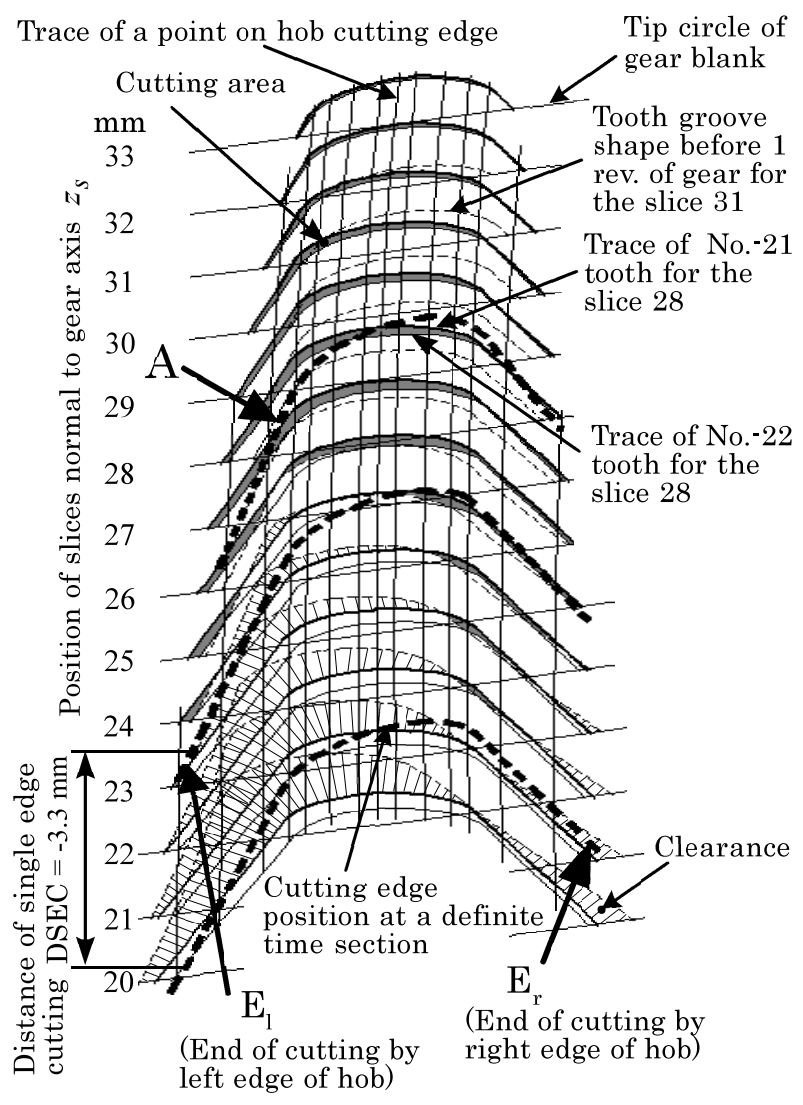

Fig. 4 Cutting area (gray area) and clearance (hatched area) on each slice of tooth groove normal to gear axis, which are expressed by traces of hob cutting edges
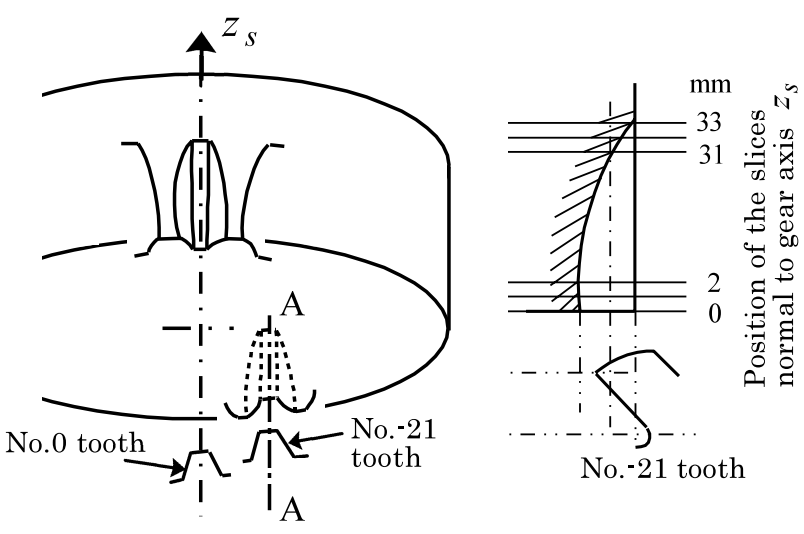

(a) View of gear and hot tooth

(b) Section A-A

Fig. 5 Definition of slices normal to gear axis

cutting edge. The gray area, where the trace of the No.-21 cutting edge is deeper than any other traces, shows the cut area with the No.-21 cutting edge. On the other hand, in the hatched area, where the tooth groove before 1 revolution of the work gear or the trace of the cutting edge of the No.-22 tooth of the hob is deeper than that of the No.-21 tooth, there is a clearance between the cutting edge of the hob and the tooth flank of the work gear.

Figure 6 shows the traces of the cutting edge of the hob on the normal-to-gear-axis slice at $z_{s}=28 \mathrm{~mm}$ and

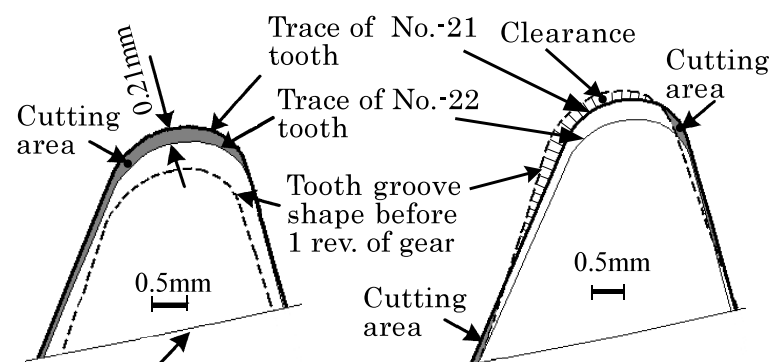

Tip circle of gear blank
(a) 2-dimensional view
$\left(z_{s}=28 \mathrm{~mm}\right)$
(b) 2-dimensional view $\left(z_{s}=24 \mathrm{~mm}\right)$

Fig. 6 Expression of cutting area and clearance between gear blank and cutting edge of No.-21 tooth of hob

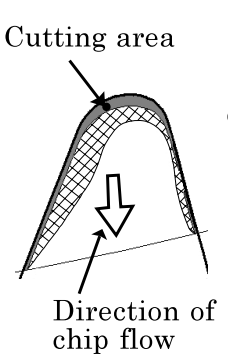

(a) Stage $\mathrm{A}$

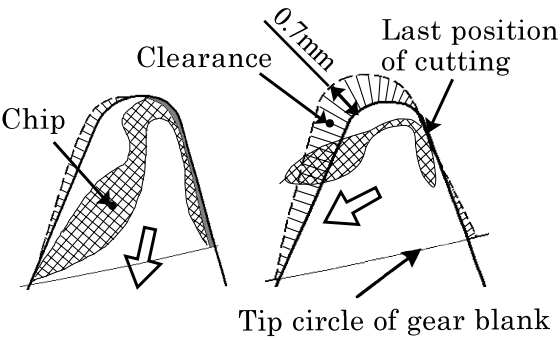

(b) Stage $\mathrm{E}_{1}$

(c) Stage $E_{r}$
Fig. 7 Difference in direction of chip flow over rake surface of hob tooth at each cutting stage

$24 \mathrm{~mm}$. The whole cutting edge around the hob tooth acts on the slice at $z_{s}=28 \mathrm{~mm}$. At the slice near $z_{s}=24 \mathrm{~mm}$, the upper left edge has finished cutting, and the cutting area is divided into 2 regions. At the end of cutting by the No.-21 hob tooth, the right cutting edge acts alone, as also shown in Fig. 4. The 2-dimensional expression on the normal-to-gear-axis slice shown in Fig. 6 enables the easy recognition of accurate size of the cutting area and clearance.

Referring again to Fig. 4 to see the chip formation process. The bold broken curve shows the cutting edge position at a definite time section. The left cutting edge of the hob begins cutting each slice earlier than the right cutting edge because of the hob set angle used in this case. The edge position $\mathrm{E}_{1}$ corresponds to the end of cutting of the left edge and $E_{r}$ to the end of cutting of the right edge. The tooth groove is cut only by the right cutting edge of the hob in the axial range from $E_{1}$ to $E_{r}(-3.3 \mathrm{~mm}$ (positive sign means that the right edge finishes cutting before the left edge)). This axial distance is defined here as DSEC (distance of single-edge cutting).

Figure 7 shows a supposed movement of the generated chip over the rake surface of the hob estimated from the result of simulation for stages $A, E_{1}$ and $E_{r}$ in Fig. 4. The whole tooth groove is cut simultaneously in Fig. 7 (a), and the chip therefore moves toward the tooth root of the hob. The tip and the left cutting edge of the hob finishes 
its work at stage $\mathrm{E}_{1}$ (cf. Fig. 4) in Fig. 7 (b) and the right cutting edge acts alone until stage $\mathrm{E}_{\mathrm{r}}$ (cf. Fig. 4) as shown in Fig. 7 (c). At stage $E_{r}$, the chip moves toward the left edge of the hob. A large part of the already generated chip can surely reach the position near the left cutting edge of the hob. On the other hand, the clearance between the left cutting edge and the tooth flank of the work gear appears between stages $A$ and $E_{1}$ (cf. Fig. 4) and it becomes larger. Under such a condition, the generated chip could intrude into the clearance, which leads to chip crush. The chipping position of the cutting edge of the hob in Fig. 1 corresponds well to the position of the clearance in Fig. 7 (c), where generated chips would intrude. This suggests a main cause of the chipping is supposed to be chip crush. Chip crush does not always occur even if there is clearance and the generated chips move towards the clearance: There would be cases where chips do not intrude into the clearance or pass through the clearance without crush. The chipping of the cutting edge is not actually observed in neighboring hob teeth of the No.-21 tooth, although there is no big difference in simulation results for the condition of chip formation and the behavior of clearance between the cases of the No.-21 hob tooth and the neighboring hob teeth. Not only clearance but also the curl of the chip and the surface conditions of the tooth flank of the work gear influence the occurrence of chip crush. It is therefore difficult to predict exactly the occurrence of chip crush. The proposed simulation, however, is able to evaluate the possibility of chip crush, which is industrially useful.

\section{Influence of Gear Dimensions on Chip Crush}

The cutting of a gear whose dimensions are shown in Table 1 (a) by the hob shown in Table 1 (b) under the cutting conditions listed in Table 1 (c) is taken as an example. Figure 8 shows the simulation result for the No.-14 tooth of the hob. The result of the investigation in the following chapter confirms that the general features of chip crush are not strongly influenced by the difference of the cutting edge of the hob. The analysis of the No.-14 cutting edge is incorporated here as representative to see the phenomena. Figure 8 (b) shows the traces of the cutting edge on the normal-to-gear-axis slice at $z_{s}=23 \mathrm{~mm}$.

The failure of the cutting edge often becomes a problem when a large chip is generated. Figure 8 (c) shows the chip conditions over the rake surface of the hob tooth before the end of cutting. The DSEC is short $(0.9 \mathrm{~mm})$ in this case although only the left cutting edge remains at work in that stage. This indicates that the time when the chip moves toward the right cutting edge of the hob tooth is short, the distance of its movement is also short. In addition, the clearance near the right cutting edge is small due to short DSEC. There is therefore little probability of chip crush under such conditions.
Table 1 Standard specifications of gear and hob, and cutting conditions

(a) Specifications of gear

\begin{tabular}{|lc|c|}
\hline Module & $m$ & 2.5 \\
\hline Pressure angle & $\alpha$ & $20^{\circ}$ \\
\hline Number of teeth & $z$ & 59 \\
\hline Helix angle & $\beta$ & $30^{\circ} \mathrm{RH}$ \\
\hline Cutting depth & & $5.875 \mathrm{~mm}$ \\
\hline Addendum modification factor & $x$ & 0 \\
\hline Outside diameter & $d_{a}$ & $175.32 \mathrm{~mm}$ \\
\hline
\end{tabular}

(b) Specifications of hob

\begin{tabular}{|lc|c|}
\hline Module & $m_{0}$ & 2.5 \\
\hline Pressure angle & $\alpha_{0}$ & $20^{\circ}$ \\
\hline Outside diameter & $z_{1}$ & $85 \mathrm{~mm}$ \\
\hline Number of threads & $4 \mathrm{RH}$ \\
\hline Lead angle & $G_{n}$ & $1.57^{\circ}$ \\
\hline Number of gashes & $0 \mathrm{~mm}$ \\
\hline Amount of protuberance & $0.90 \mathrm{~mm}$ \\
\hline Radius of top corner & \\
\hline
\end{tabular}

(c) Cutting conditions

\begin{tabular}{|c|c|}
\hline Feed of table revolution & $2 \mathrm{~mm} / \mathrm{rev}$. \\
\hline Direction of feed & Climb \\
\hline Hob set angle & $22.43^{\circ}$ \\
\hline
\end{tabular}

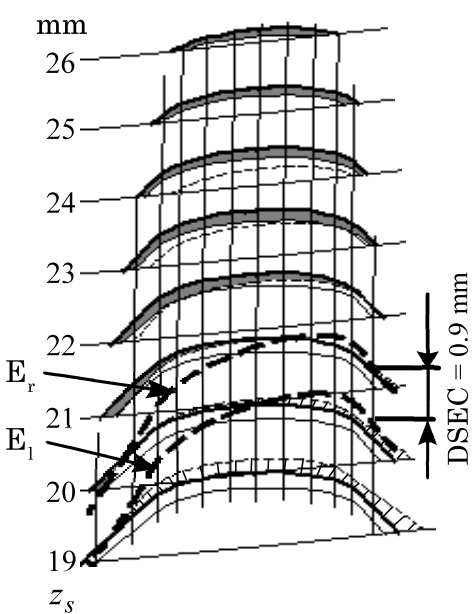

(a) 3-dimensional view

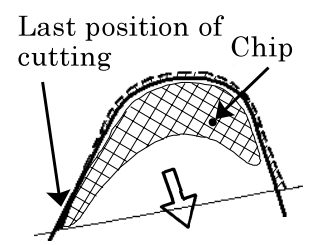

(c) Chip flow over rake surface

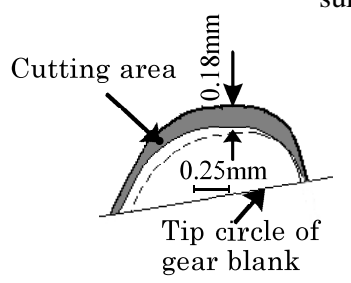

(b) 2-dimensional view $\left(z_{s}=23 \mathrm{~mm}\right)$

Fig. 8 Cutting area, clearance and chip flow over rake surface for No.-14 tooth under standard conditions

\subsection{Influence of addendum modification factor}

Figure 9 shows the simulation result for the hobbing of the gear whose addendum modification factor $x$ is changed from 0 to 0.5 or -0.5 . The outside diameter of the work gear is changed from that of Fig. 8 according to 

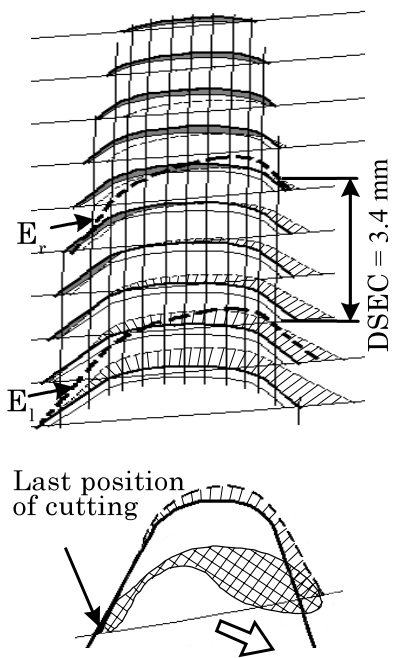

(a) $x=0.5$

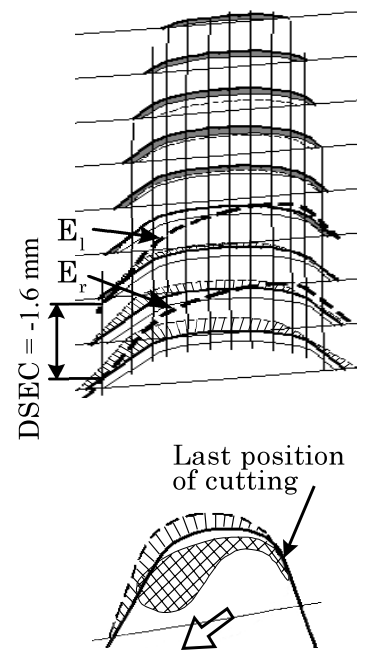

(b) $x=-0.5$

Fig. 9 Influence of addendum modification factor $x$ of work gear

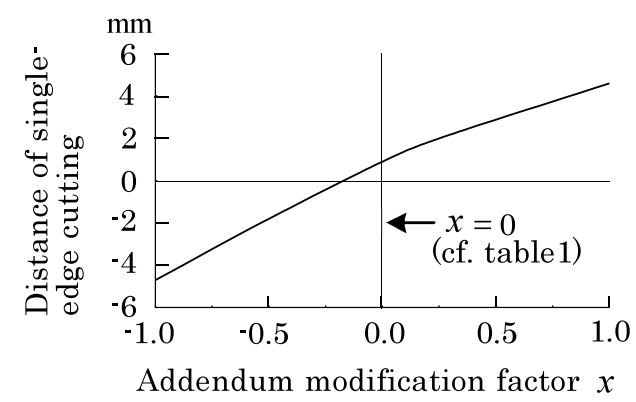

Fig. 10 Effect of addendum modification factor of work gear on DSEC

the change in addendum modification factor $x$. For gear of $x=0.5$, the DSEC becomes longer $(3.4 \mathrm{~mm})$ and the clearance between the cutting edge and the tooth flank of the work gear becomes larger. The lower panel of Fig. 9(a) shows the condition of chip formation over the rake surface of the hob tooth. Chips move toward the clearance near the right cutting edge because the left cutting edge acts alone for a long time. These conditions imply that there is a high probability of chip crush.

DSEC is $-1.6 \mathrm{~mm}$ for gear of $x=-0.5$ (see Fig. 9 (b)). This indicates that only the right cutting edge acts at the end of cutting, which is opposite that of the previous examples. A large clearance appears near the left cutting edge and chips can crush between the cutting edge and the tooth flank of the work gear.

A large absolute value of DSEC implies a large clearance and a large movement of chips over the rake surface of the hob tooth, that is, a high probability of chip crush exists. DSEC can therefore be taken as an index for evaluating the probability of chip crush.

DSEC increases according to increase in addendum modification factor as shown in Fig. 10. DSEC changes from negative to positive. This means that whether the cut-

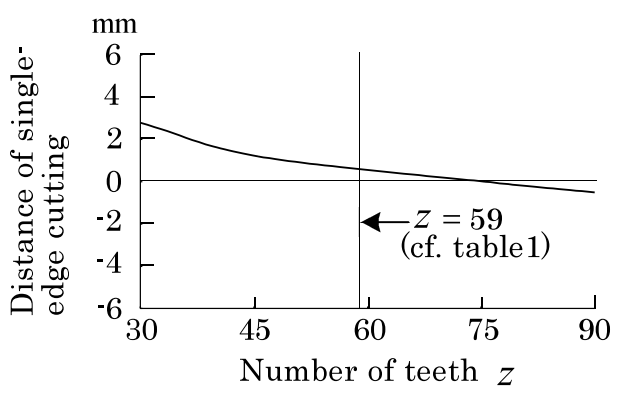

Fig. 11 Effect of number of teeth on DSEC

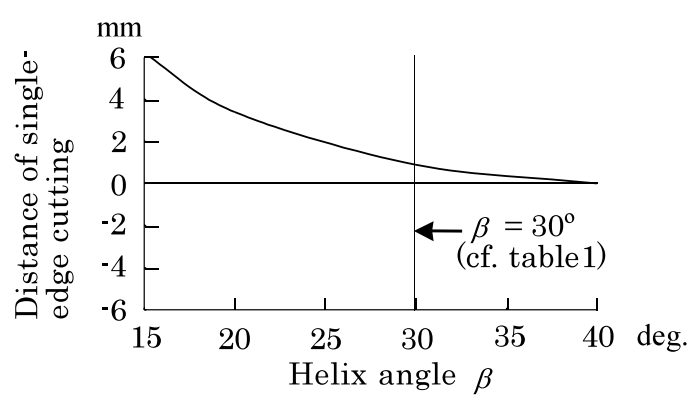

Fig. 12 Effect of helix angle of work gear on DSEC

ting edge at the end of cutting is left or right depends on the addendum modification factor of work gears. DSEC takes 0 at $x=-0.2$ in this case. That is, the left and right cutting edges finish cutting at the same time and there is little probability of chip crush under such condition. The change in the addendum modification factor from 0.2 increases the probability of chip crush. In actual cases, a small difference in gear dimensions results in the difference in the difficulty of dry hobbing. This fact is partly confirmed by the simulation result shown in Fig. 10.

\subsection{Influence of tooth number, helix angle and pressure angle}

Figure 11 shows the influence of the tooth number of the gear on DSEC. DSEC is approximately 0 at large tooth number but changes at small tooth number. Figure 12 shows the result of the investigation concerning helix angle (The outside diameter of work gear is adjusted simultaneously). It is clear that DSEC approaches 0 when helix angle increases. This suggests that there is rather high probability of chip crush in the hobbing of gears with a small helix angle. The result of the investigation concerning pressure angle is shown in Fig. 13. There is no considerable influence of pressure angle on DSEC.

\section{Influence of Hob Dimension and Cutting Condi- tions}

Figure 14 shows the relationship between the number of threads of the hob and DSEC, where the number of threads is changed from the value shown in Table 1 and parameters such as hob set angle and hob lead angle are adjusted according to the change for the simulation. The probability of chip crush is very low for a hob with 1 or 


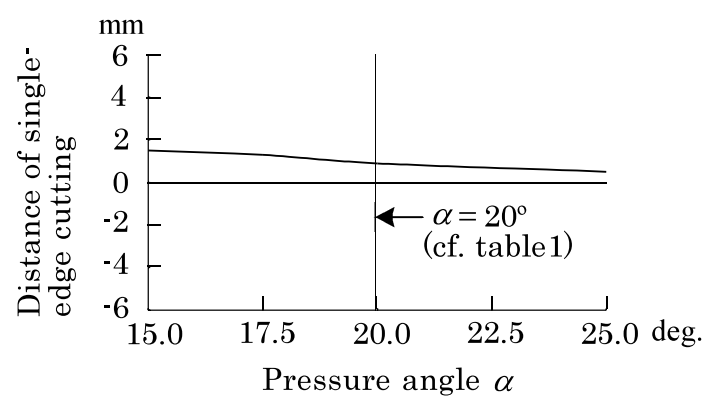

Fig. 13 Effect of pressure angle of work gear on DSEC

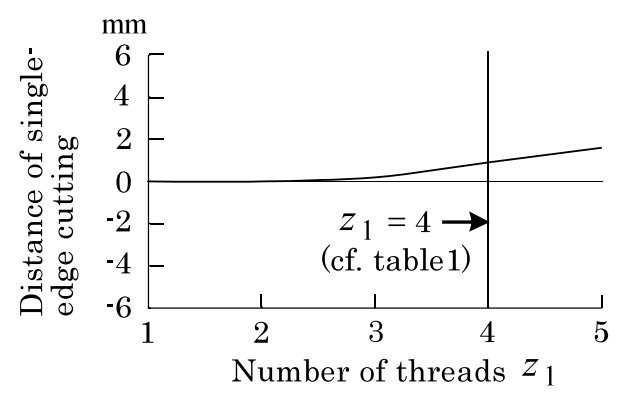

Fig. 14 Effect of number of threads of hob on DSEC

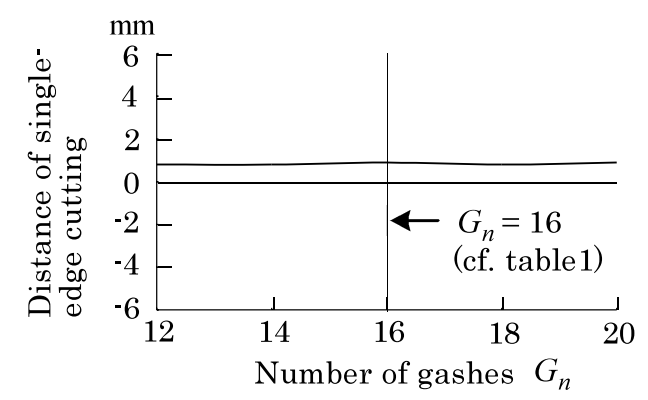

Fig. 15 Effect of number of gashes of hob on DSEC

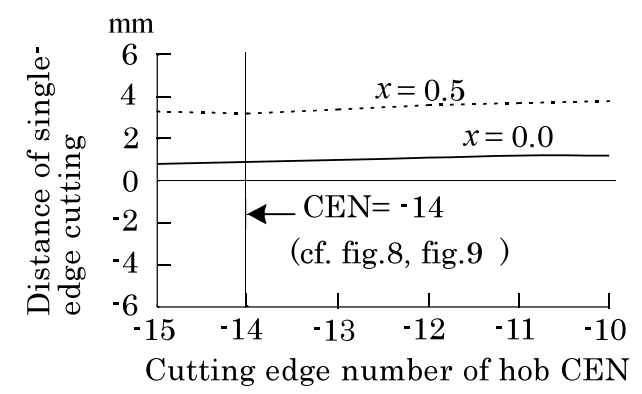

Fig. 16 DSEC at different cutting edges of hob

2 threads because the DSEC is near 0 but the probability of chip crush increases when a hob with a large number of threads is used.

The result concerning the number of gashes of the hob is shown in Fig. 15. It is clarified that the number of gashes has little influence on the probability of chip crush.

Figure 16 shows the change in DSEC at different cutting edges of the hob, where the work gears of addendum modification factors 0 and 0.5 are taken as examples. It is clear that DSEC changes hardly for each different cutting

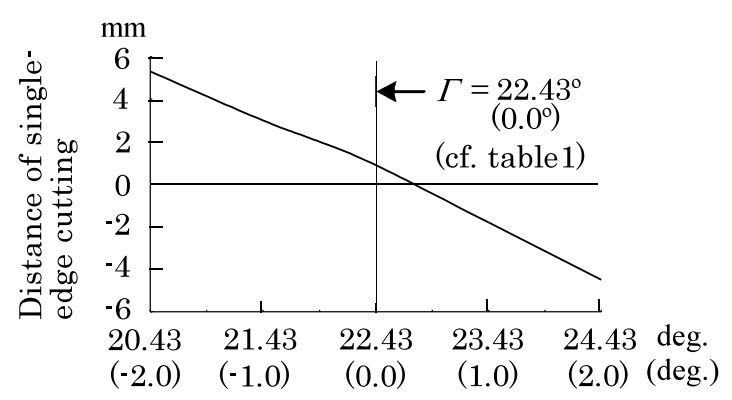

Hob set angle $\Gamma$

(Amount of modification)

Fig. 17 Effect of hob set angle on DSEC

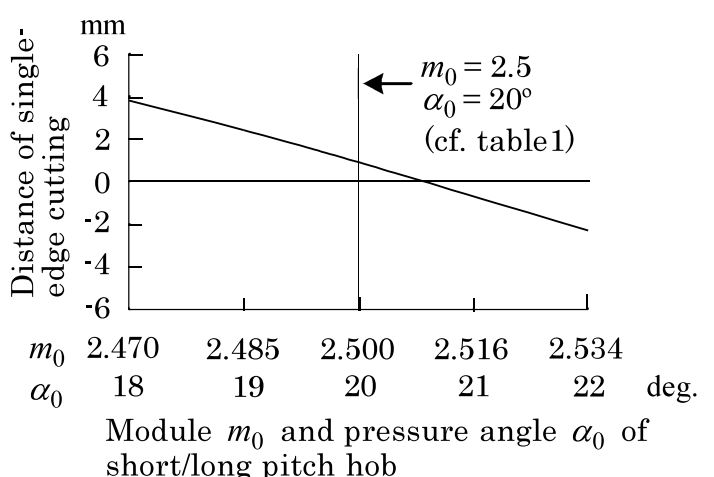

Fig. 18 Effect of short/long pitch hob on DSEC

edge of the hob. This means any cutting edge of minus large absolute number can be taken for the analysis of the probability of chip crush.

Figure 17 shows the influence of hob set angle. The value in parenthesis shows the amount of modification of hob set angle from the standard value. DSEC markedly changes with the change in hob set angle. The adjustment of hob set angle is considered to be effective for changing the conditions of chip formation and the behavior of the clearance.

A short/long pitch hob has different pressure angle and module but it has the same normal pitch compared with the original standard hob. The same gear can be cut by such a short/long pitch hob. Figure 18 shows the change in DSEC by such a short/long pitch hob under a fixed normal pitch. DSEC decreases with the increase in module and pressure angle. This indicates that the short/long pitch hob can change the conditions of chip formation and the behavior of the clearance to prevent chip crush.

For left-hand hobs, DSEC has a sign opposite to the case of right-hand hobs.

\section{Method to Solve Problems Due to Chip Crush}

Problems concerning chip crush often occur in the trial cut stage or in the early stage of mass production. These problems must be solved by changing parameters unrelated to gear dimension because gear dimension can- 

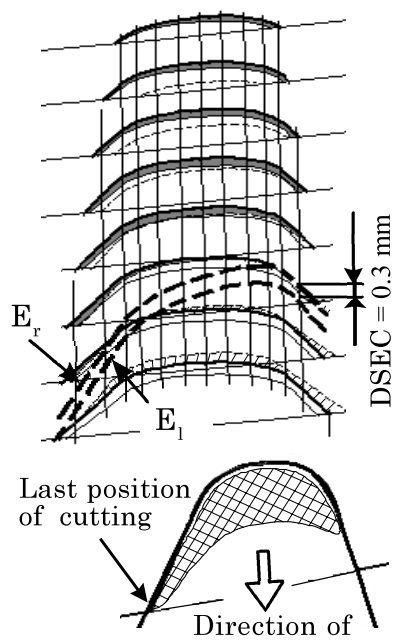

chip flow

(a) $\quad \Gamma=23.63^{\circ}$

$\left(+1.2^{\circ}\right.$ correction $)$
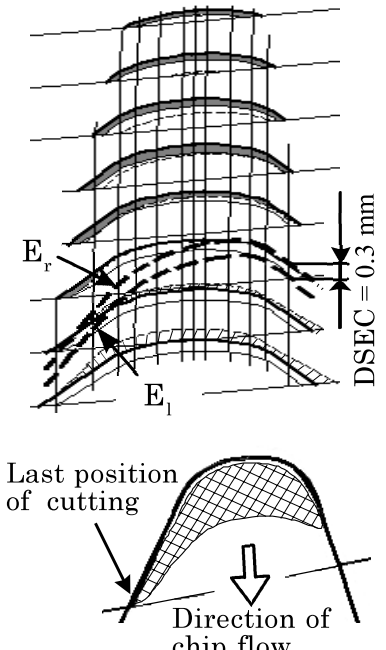

(b) $m_{0}=2.535, \alpha_{0}=22.07^{\circ}$ (cf. Table 1)

Fig. 19 Improvement of cutting conditions by modifying hob set angle or applying long pitch hob $\left(m_{0}\right.$ : hob module, $\alpha_{0}$ : hob pressure angle)

not be changed at such stages. The condition shown in Fig. 9(a) is taken here as an example and the problem due to chip crush at the right cutting edge is to be solved. DSEC is long $(3.4 \mathrm{~mm})$ under the cutting conditions and must be decreased. An increase in hob set angle leads to a decrease in DSEC, as shown in Fig. 17. DSEC can also be decreased by applying a long pitch hob of a larger module and pressure angle. Figure 19 (a) shows the simulation result for the +1.2 degree modification of the hob set angle and Fig. 19 (b) shows the case of the incorporation of the long pitch hob of module 2.535 and 22.07 degrees pressure angle. It is clear that the DSEC approaches almost 0 by the proposed methods and probability of chip crush is supposed to be lower.

\section{Application to Actual Problems of Hobbing}

\subsection{Failure of left cutting edge of right-hand hob (example 1)}

The chipping shown in Fig. 1 is taken here as the first example, where simulation results are shown in Fig. 4. Figure 20 shows the simulation result, where hob set angle is changed by -0.75 degrees. The DSEC changes from $-3.3 \mathrm{~mm}$ (see Fig. 4) to $0.2 \mathrm{~mm}$. This countermeasure was applied to the actual case of mass production of automotive gears and the chipping problem was completely solved.

\subsection{Failure of left cutting edge of left-hand hob (ex- ample 2)}

Figure 21 shows the chipping of the cutting edge of the No.-26 tooth of the hob observed when the left-hand helical gear (module 2, pressure angle 20 degrees, number of teeth 31 , helix angle 35 degrees and addendum mod-

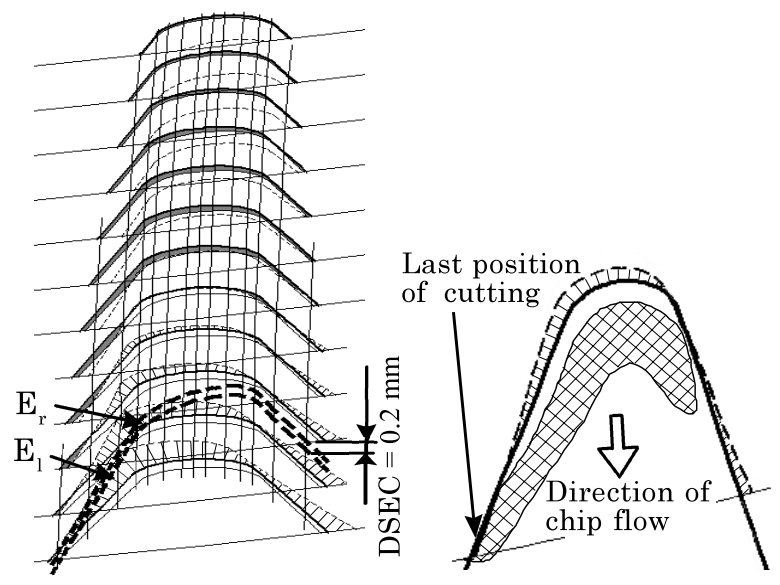

Fig. 20 Simulation result when countermeasure against hob chipping is applied by hob set angle modification of $-0.75 \mathrm{deg}$.

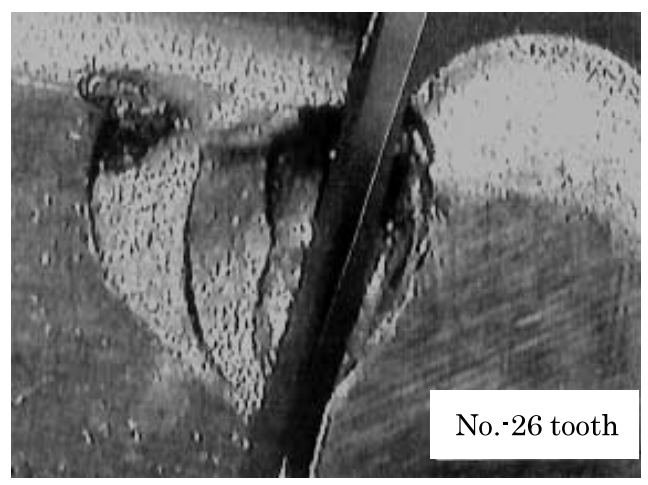

Fig. 21 Chipping of cutting edge of carbide hob (example 2)

ification factor 0.33) is dry-cut using a left-hand carbide hob (3 threads) at a climb feed of $2.5 \mathrm{~mm} / \mathrm{rev}$. The same chipping failure occurred repeatedly at the same position on the hob tooth. Figure 22 (a) shows the simulation result corresponding to the hobbing conditions shown in Fig. 21. Chip crush at the left edge of the hob is supposed to be the cause of chipping because the DSEC is long $(-3.3 \mathrm{~mm})$.

Figure 22 (b) shows the result of the simulation, where the hob set angle is modified by +1 degree. The absolute value of DSEC is decreased to $0.2 \mathrm{~mm}$. In actual mass production, this correction of hob set angle is given. Then the chipping failure of the cutting edge of the hob disappeared.

\subsection{Failure of right cutting edge of right-hand hob (example 3) $)^{(5)}$}

Figure 23 shows the chipping of the No.-14 cutting edge of the hob observed when the right-hand helical gear (module 2.4, pressure angle 20 degrees, number of teeth 69 , helix angle 30 degrees and addendum modification factor 1.0) is dry-cut using a right-hand carbide hob (4 threads) at a climb feed of $2 \mathrm{~mm} / \mathrm{rev}$. Figure 24 (a) shows its simulation result. Chip crush is regarded as a main cause of the chipping shown in Fig. 23 because the DSEC 

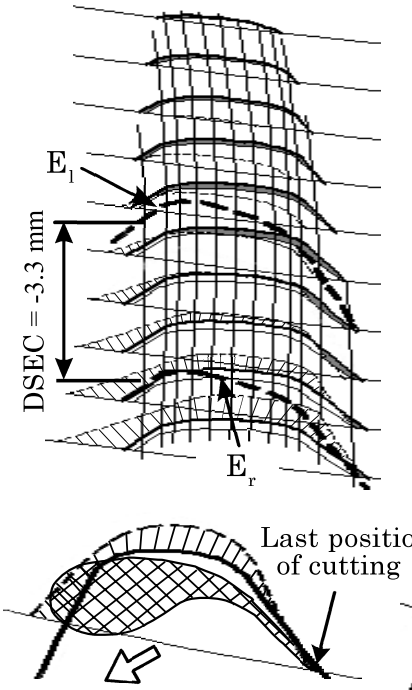

Direction of chip flow

(a) No correction

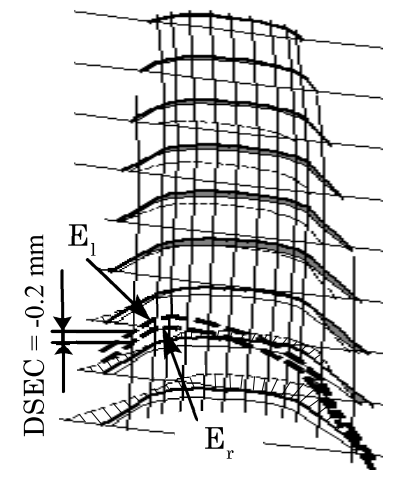

Last position

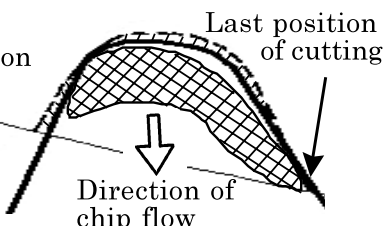

chip flow

(b) Hob set angle correction of +1 deg.

Fig. 22 Simulation result of No.-26 tooth of example 2, where hob set angle modification of +1 deg. is given

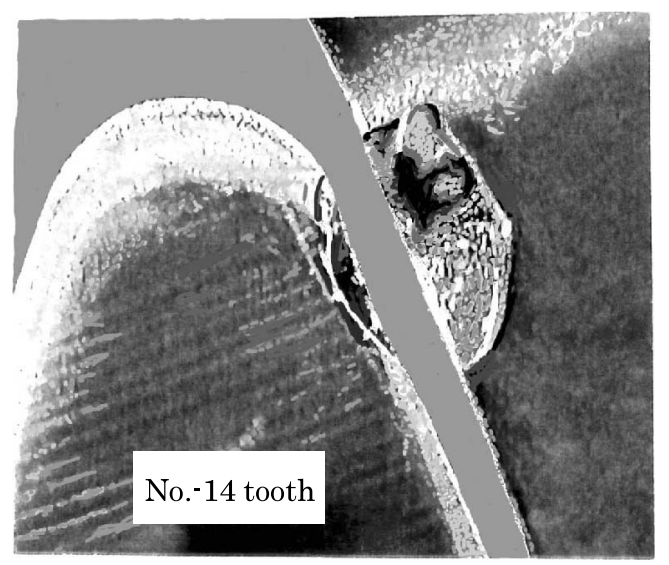

Fig. 23 Chipping of cutting edge of carbide hob (example 3)

\section{is long $(4.0 \mathrm{~mm})$.}

The DSEC can be decreased to $-0.2 \mathrm{~mm}$ by modifying hob set angle by +1.5 degrees, as shown in Fig. 24 (b). The clearance at the right edge becomes smaller as well. This remedy was applied to an actual case and the problem of chipping failure was solved.

The above-presented actual cases confirm that the proposed remedies are useful in preventing hob failure due to chip crush.

\section{Conclusions}

Failures of the cutting edge of a hob or problems about the surface quality of the tooth flank of a manufactured gear are serious problems in dry hobbing. These problems are usually caused by chip crush between the cutting edge of a hob and the tooth flank of a work gear. In

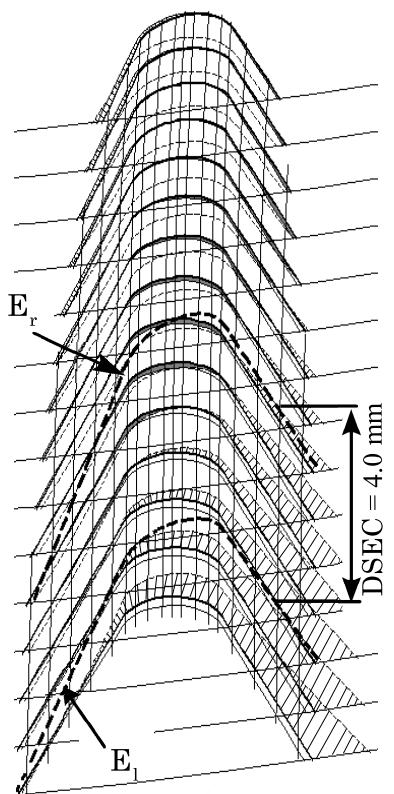

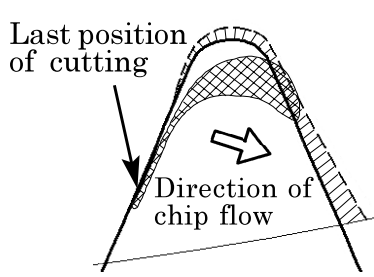

(a) No correction
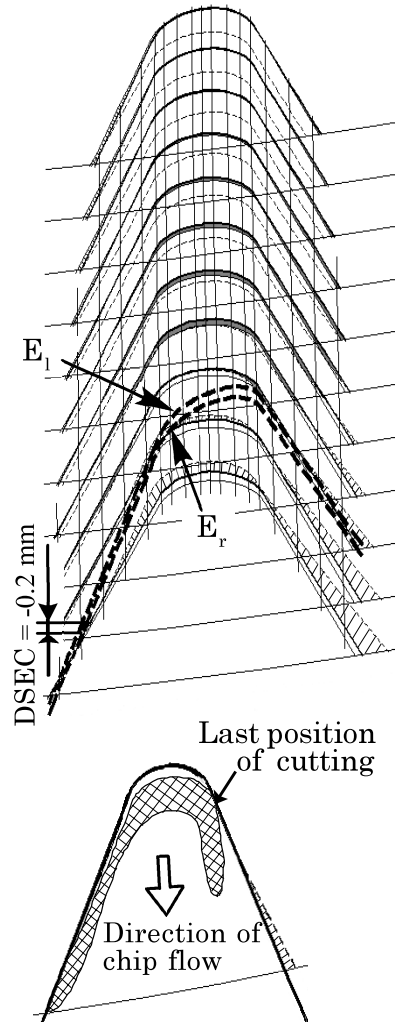

(b) Hob set angle correction of $+1.5 \mathrm{deg}$.
Fig. 24 Simulation result of No.-14 tooth of example 3, where hob set angle modification of $+1.5 \mathrm{deg}$. is given

this report, the behavior of chip formation and the changing state of clearance between the cutting edge of a hob and the tooth flank of a work gear are investigated by the proposed simulation method to develop a method of preventing chip crush. The following conclusions are drawn.

(1) Unnaturally bent chips are found in the pile of chips by dry hobbing, in which the chipping of cutting edge of the hob occurs. Such chips are probably crushed between the cutting edge of a hob and the tooth flank of a work gear.

(2) The position of chipping of the cutting edge of a hob corresponds very well to the position of chip crush anticipated from the simulation result. Thus, the possibility of chip crush can be evaluated using the proposed simulation.

( 3 ) DSEC, that is, axial distance concerning a work gear where only a single edge cuts a tooth groove, can be taken as an index for evaluating the possibility of chip crush: a large absolute value of DSEC means a high probability of chip crush.

(4) Addendum modification factor, helix angle, the number of teeth of a gear, hob set angle, and short/long pitch hob have a strong influence on DSEC. In contrast, 
pressure angle, the number of gashes, and the difference in the cutting edge of hob have little influence. A hob with a large number of threads, e.g., 3 or more, results in larger DSEC.

(5) A solution to the problems of chipping of the cutting edge of a hob due to chip crush is to lower DSEC. For that we propose the following: (1) changing the hob set angle, (2) use of a short/long pitch hob of a modified module and pressure angle. Such methods make it possible to prevent chip crush without changing the dimensions of the manufactured gears. The method has been applied to solving actual chipping problems in the mass production of gears and successful results have been accomplished.

\section{References}

(1) Sakuragi, I., Kobayashi, M., Hakamata, N., Kawata, M., Sumi, M. and Yonekura, M., Carbide Hobbing Technology for Automotive Gears, Trans. Jpn. Soc. Mech. Eng., (in Japanese), Vol.67, No.655, C (2001), pp.811-816.

( 2 ) Research Report, (in Japanese), (2000), Japan Gear Manufacturers Association.
( 3 ) Sakuragi, I., Ochi, Y., Kawata, M., Nishioka, M., Yonekura, M. and Tsumura, K., Countermeasures of Inclusion of Chips on Gear Tooth Surface during HighSpeed Dry Hobbing, Prepr. of Jpn. Soc. Mech. Eng., (in Japanese), No.01-1 (2001), pp.147-148.

( 4 ) Sumi, M., Kubo, A. and Komori, M., Hob Cutting Simulation for Analysis of Chip Crush between Cutting Edge of Hob and Tooth Flank of Gear, Trans. Jpn. Soc. Mech. Eng., (in Japanese), Vol.69, No.681, C (2003), pp.1388-1395.

( 5 ) Sakuragi, I., Yonekura, M. and Sumi, M., A Research on High-Speed Hobbing with Carbide Multiple-Thread Hob (For Countermeasures against Chipping on the Cutting Edge), Prepr. of Jpn. Soc. Mech. Eng., (in Japanese), No.98-1 (1998), pp.577-578.

( 6 ) Terashima, K. and Ueno, T., Trans. Jpn. Soc. Mech. Eng., (in Japanese), Vol.43, No.373, C (1977), pp.3535-3546.

( 7 ) Asakura, S., Aoyama, N., Asakura, S. and Fukuta, K., The Technology of High Performance Gear Cutting with Carbide Hob in Mass Production, Prepr. of Jpn. Soc. Mech. Eng., (in Japanese), No.98-1 (1998), pp.579-580. 